\title{
Active suppression of acoustic radiation from impulsively excited structures
}

\author{
William T. Baumann \\ Interdisciplinary Center for Applied Mathematics, Smart Materials and Structures Laboratory, Bradley \\ Department of Electrical Engineering, Virginia Polytechnic Institute and State University, Blacksburg, \\ Virginia 24061 \\ William R. Saunders and Harry H. Robertshaw \\ Interdisciplinary Center for Applied Mathematics, Smart Materials and Structures Laboratory, Department \\ of Mechanical Engineering, Virginia Polytechnic Institute and State University, Blacksburg, Virginia 24061
}

(Received 12 July 1990; revised 1 March 1991; accepted 2 August 1991)

\begin{abstract}
The objective is to use active control to suppress the acoustic energy that is radiated to the far field from a structure that has been excited by a short-duration pulse. The problem is constrained by the assumption that the far-field pressure cannot be directly measured. Therefore, a method is developed for estimating the total radiated energy from measurements on the structure. Using this estimate as a cost function, a feedback controller is designed using linear quadratic regulator theory to minimize the cost. Computer simulations of a clampedclamped beam show that there is appreciable difference in the total radiated energy between a system with a controller designed to suppress vibrations of the structure and a system with a controller that takes into account the coupling of these vibrations to the surrounding fluid. The results of this work provide a framework for a general, model-based method for actively suppressing transient structural acoustic radiation that can also be applied to steady, narrow, or broadband disturbances.
\end{abstract}

PACS numbers: $43.40 . \mathrm{Vn}, 43.40 . \mathrm{Rj}$

\section{INTRODUCTION}

Active structural acoustic control, using modern control methods, is introduced here as a natural extension to active vibration control. Numerous investigations, both theoretical and experimental, have been conducted on active control of elastic structures. ${ }^{1-5}$ Such control methods are model based, typically posed in state-space forms, and rely on state or output feedback to introduce additional damping to the structure. The controllers may be designed for suppression of transient response (induced by initial conditions), or rejection of a continuous disturbance force on the structure. For vibration control, it is typical to choose modal coordinates as the basis for the state vector. However, several authors have discussed vibration control from a wave propagation perspective. ${ }^{6-8}$ It is anticipated that either basis would allow a formulation of the structural acoustic control problem, but we choose to work with the modal control approach.

Recently, there has been some interest in the active control of sound radiation from elastic structures using force inputs. Fuller ${ }^{9,10}$ demonstrated, both experimentally and analytically, that significant reduction in far-field radiated pressure was possible by applying control forces directly to a plate. By minimizing a radiated power cost function, the optimal control was derived which caused reduction in farfield pressure. The experimental work by Fuller used acoustic pressure sensors in the far field to generate a radiated power cost function that was minimized using electrodynamic actuators on a circular plate. The control experiment used an adaptive algorithm, which has been discussed in Refs. 11 and 12. A state-space method for active control of sound radiation ${ }^{13}$ by Meirovitch uses a standard LQR controller design for vibration control, with observation of the resulting far-field radiated pressures to verify the effectiveness of the control. In the following, we also discuss a statespace method but the acoustic radiation dynamics are implemented directly into the controller. The concept of this controller design for structural acoustic control was discussed briefly in an overview paper by Fuller et al. ${ }^{14}$

In this paper, we distinguish the structural acoustic control objective as a requirement to suppress only those surface velocity vectors that are efficiently coupled to the far field. It will be shown that this new requirement necessarily increases the order of the controller, but provides a significant reduction in radiated acoustic energy compared to traditional active vibration control. Additionally, this objective may actually reduce the number of control actuators required. It is intuitive that control effort need not be expended on inefficiently radiating modes. The controller design discussed in this paper ensures that the dominant radiation modes of a structure will be actively damped most quickly in response to an impulsive loading.

We consider a structure (immersed in a light fluid, such that $\rho_{0} / \rho_{m} \ll 1$, where $\rho_{0}$ is the fluid density and $\rho_{m}$ is the mass density of the structure) that has been excited by a mechanical impulse. The objective is to suppress the acoustic energy resulting from the pulsed input to the structure that is radiated to the far field. The problem is constrained by the 
assumption that the far-field pressure cannot be directly measured. Therefore, a method is developed for estimating the total radiated energy from measurements on the structure. Using this estimate as a cost function, a feedback controller is designed using linear quadratic regulator theory to minimize the cost. It will be shown that the cost function is practically equal to that used by Fuller, as discussed previously. However, the state-space formulation allows a timedomain optimal control that is not available in the aforementioned work.

Computer simulations of a clamped-clamped beam show an appreciable difference in the total radiated energy between a system with a controller designed to suppress vibrations of the beam and a system with a controller that takes into account the coupling of these vibrations to the surrounding fluid. Numerical results will be presented after the formulation of the structural acoustic control law.

\section{ESTIMATING TOTAL RADIATED ENERGY}

Consider a flexible structure that can be accurately modeled by a first-order ordinary differential equation of the form

$$
\dot{w}=A w+B u+L v,
$$

where $w \in R^{2 n}$ is the state of the system, $u \in R^{m}$ is a vector of actuator inputs that can be used to control the structure, and $v \in R^{p}$ is the pulsed disturbance input. The approximate velocity of the structure at position $x$ and time $t$ is given by

$$
v(x, t)=\sum_{i=1}^{N} \Phi_{i}(x) w_{2 i}(t)
$$

We incorporate a standard modal model in the examples; however, the $\Phi_{i}$ functions need not be modes and can be any set of functions, such as finite element discretizations, that can approximate the actual velocity via Eq. (2) to the desired accuracy.

The pressure in the far field at the point $(R, \theta, \phi)$ in a fixed spherical coordinate system, due to a harmonic input of frequency $\omega$, can be computed from the Rayleigh integral as

$$
p(t)=\frac{j \omega \rho}{2 \pi} \int_{S} \frac{v(S, t) e^{j k\left(R-r_{s}\right)}}{\left(R-r_{s}\right)} d S .
$$

The pressure resulting from the velocity distribution associated with the $i$ th spatial function on the structure

$$
v(x, t)=w_{2 i}(t) \Phi_{i}(x),
$$

where

$$
w_{2 i}(t)=w_{2 i} e^{j \omega t}
$$

can be written as

$$
p(t)=w_{2 i}\left(\frac{j \omega \rho e^{j \omega t}}{2 \pi} \int_{S} \Phi_{i}(S) e^{-j k r_{s}} d S\right) \frac{e^{j k R}}{R},
$$

where the factor of $e^{j k R}$ accounts for the propagation delay, the factor of $R$ in the denominator accounts for the spherical spreading loss, and $S$ is the surface area domain of the radiator.
From a system-theoretic point of view, we can consider the input of the system to be the time variation of the $i$ th mode, $w_{2 i}(t)$, and the output to be the pressure, $p(t)$, at a given point $(R, \theta, \phi)$. For a harmonic input of the form in Eq. (5), Eq. (6) shows that the output can be written as

$$
p(t)=\hat{h}(\omega) w_{2 i} e^{j \omega t},
$$

which is in the form of the steady-state response of a linear system with transfer function $\hat{h}(\omega)$, where $h(\omega)$ is the Fourier transform of the system impulse response $h(t)$. (In the remainder of the paper, the Fourier transform of a variable will be denoted by a carat, . ) For a stable system, we can characterize the transfer function from steady-state considerations. However, knowledge of the transfer function completely specifies the system since it is equivalent to knowing the impulse response. By building (i.e., specifying a differential equation for) a filter having the desired transfer function, the response of the system to transient, as well as steady-state, excitations can be computed by applying the desired input waveform to the filter and observing the output. Note that filtering the input in the time domain is a causal operation, and thus can be implemented in real time. The output pressure could also be computed by taking the Fourier transform of the input and then taking the inverse Fourier transform of the product $\hat{h}(\omega) \hat{w}_{2 i}(\omega)$, but this cannot be done causally.

We will write the transfer function between the time variation of the velocity, $w_{2 i}(t)$, of the $i$ th spatial function $\Phi_{i}$ and the pressure in the far field at the point $(R, \theta, \phi)$ as

$$
\hat{p}_{i}(R, \theta, \phi, \omega) / \hat{w}_{2 i}(\omega)=\hat{h}_{i}(\theta, \phi, \omega) e^{j k R} / R,
$$

where the spatial dependence has been included explicitly and, for notational convenience, the delay and loss terms of the transfer function have been written separately.

Before proceeding, we define the characteristics of the input and output signals. First, it is assumed that the time duration of each signal is such that

$$
w_{2 i}(t)=p_{i}(t)=0, \quad t<t_{0},
$$

where $t_{0}$ corresponds to the application of the impulse $\delta\left(t-t_{0}\right)$. Second, we view the resulting radiated pressure as an energy signal with energy proportional to the integral of the signal squared. By the acoustic far-field assumption, the proportionality constant (between pressure and velocity) will be the characteristic impedance $\rho c$. Note that the relationship $p=(\rho c) v$ holds for instantaneous signals, as well as steady-state signals.

Using the real-valued pressure and fixing $R$, the total energy per unit area radiated into the far field in direction $(\theta, \phi)$ is given by the integration of the instantaneous power per unit area with respect to time, that is

$$
\frac{1}{\rho c} \int_{0}^{\infty}\left(\sum_{i=1}^{N} p_{i}(R, \theta, \phi, t)\right)^{2} d t
$$

Comparing Eq. (10) to Eq. (11) of Mann et al., ${ }^{15}$ we recognize the expression as a summation of the instantaneous intensity for some spatial segment over time.

By Parseval's theorem, this may be expressed as

$$
\frac{1}{\pi \rho c} \int_{0}^{\infty}\left|\sum_{i=1}^{N} \hat{p}_{i}(R, \theta, \phi, \omega)\right|^{2} d \omega
$$




$$
\begin{aligned}
= & \frac{1}{\pi \rho c R^{2}} \int_{0}^{\infty}|H(\theta, \phi, \omega) W(\omega)|^{2} d \omega \\
= & \frac{1}{\pi \rho c R^{2}} \int_{0}^{\infty} W^{T_{*}}(\omega) H^{T_{*}}(\theta, \phi, \omega) H(\theta, \phi, \omega) \\
& \times W(\omega) d \omega,
\end{aligned}
$$

where

$$
W=\left[\begin{array}{c}
\hat{w}_{2} \\
\hat{w}_{4} \\
\vdots \\
\hat{w}_{2 n}
\end{array}\right] ; \quad H=\left[\hat{h}_{1}, \hat{h}_{2}, \ldots, \hat{h}_{n}\right] .
$$

For the above, the complex conjugate is denoted by *, and the units of Eq. (11) are energy per area $\left(\mathrm{J} / \mathrm{m}^{2}\right)$ as expected.

To compute the total radiated energy, integrate Eq. (13) over the surface of a sphere of radius $R$ such that $k R \gg 1$. This yields

$$
\int_{0}^{\infty} W^{T_{*}}(\omega)[M(\omega)] W(\omega) d \omega .
$$

where

$M(\omega)=\frac{1}{\pi \rho c} \int_{0}^{2 \pi} \int_{0}^{\pi} H^{T_{*}}(\theta, \phi, \omega) H(\theta, \phi, \omega) \sin \theta d \theta d \phi$,

where $M(\omega)$ has units corresponding to those of radiation resistance $(\mathrm{kg} / \mathrm{s})$. Thus the diagonal entries represent, up to a constant term, the self-radiation efficiencies of the "modes" $\Phi_{i}$. The off-diagonal terms similarly are the mutual-radiation efficiencies. A discussion of the mutual terms in the matrix is given later.

It is possible to approximately factor the matrix in Eq. (16), to any desired accuracy, as

$$
M(\omega) \approx G^{T_{*}}(\omega) G(\omega) .
$$

To see this, note that $H^{*}(\theta, \phi, \omega)=H(\theta, \phi,-\omega)$ and that $M(\omega)$ can be written as

$$
\frac{1}{\pi \rho c} \int_{0}^{2 \pi} \int_{0}^{\pi} H^{T}(\theta, \phi,-\omega) H(\theta, \phi, \omega) \sin \theta d \theta d \phi .
$$

In the Laplace transform domain, consider the matrix

$$
M(s)=\frac{1}{\pi \rho c} \int_{0}^{2 \pi} \int_{0}^{\pi} H^{T}(\theta, \phi,-s) H(\theta, \phi, s) \sin \theta d \theta d \phi,
$$

where $H(\theta, \phi, s)$ is the Laplace transform associated with $H(\theta, \phi, \omega)$ and $M(s)$ agrees with Eq. (18) when $s=j \omega$. The matrix $\boldsymbol{M}(s)$ is real $\left[\boldsymbol{M}^{*}(s)=\boldsymbol{M}\left(s^{*}\right)\right]$, paraconjugate Hermitian $\left[M^{T_{*}}(s)=M\left(-s^{*}\right)\right]$, and non-negative on the real-frequency axis $s=j \omega \quad\left(b^{T_{*}} M(j \omega) b \geqslant 0, \forall b \in \mathscr{C}^{n \times 1}\right)$. This positive semidefiniteness of the matrix is implied by its physical interpretation as a radiated energy operator. Approximating $M(s)$ to any desired accuracy by a rational matrix $\widetilde{M}(s)$ allows us to write

$$
\widetilde{M}(s)=G^{T}(-s) G(s),
$$

where $G(s)$ is a real, rational matrix that is analytic in $\operatorname{Re}(s)>0$. Substituting $s=j \omega$ produces the desired factori- zation. (A formal proof that the spectral factorization of the matrix quantity exists was presented by Youla. ${ }^{16} \mathrm{~A}$ constructive proof in state-space form can be found in Francis. ${ }^{17}$ ) In general, $M(s)$ will be of normal rank $n$ and $G(s)$ will be an $n \times n$ matrix. For certain degenerate cases, it may happen that $M(s)$ will be of normal rank $r<n$, in which case $G(s)$ will be of dimension $r \times n$. In the following, it will be assumed that $M$ is not degenerate, but the degenerate case follows immediately.

The total radiated energy may be expressed as

$$
\Pi=\int_{0}^{\infty} W^{T_{*}}(\omega) G^{T_{*}}(\omega) G(\omega) W(\omega) d \omega
$$

and by Parseval's theorem this is equivalent to

$$
\Pi=\int_{0}^{\infty} z^{T}(t) z(t) d t,
$$

where $z(t)=\mathscr{L}^{-1}[G(s) W(s)]$. That is, $z \in R^{n}$ is the result of passing the vector of modal (velocity) amplitudes $w_{2 i}$ through a filter with transfer function $G(s)$. Thus, by computing the filter transfer function $G(s)$ to the desired accuracy and measuring the modal amplitudes, it is possible to approximate the total far-field radiated power by Eq. (22).

It should be noted that the "radiation filter," $G(s)$, is a causal operator on the input (modal velocity) signal. This is a consequence of the fact that the spectral factorization produces a $G(s)$ with no poles in $\operatorname{Re}(s)>0$. Interpreting $G(s)$ as a bilateral Laplace transform, which has a region of convergence including the $j \omega$ axis, the absence of right-half plane poles means that the inverse transform, which is the impulse response of the filter, is zero for $t<0$ (Ref. 18). Hence, the filter is causal. This is to be expected as the factorization was originally employed to solve the Weiner-Hopf problem, the goal of which was to generate causal optimal filters (see Ref. 19). Thus, we have designed a physically realizable frequency/spatial filter to produce system states that can be used to generate a radiated power cost function. The squared magnitude of the state $z(t)$ can be viewed as the energy radiated through the given sphere at time $t+R / c$ due to the velocity field up to time $t$. The transfer function $G(s)$ does not contain a propagation delay, $e^{j k R}$, as this term canceled out in Eq. (13).

Note that the form of the radiation resistance matrix, $M(s)$, determines whether the power radiated by the various modes is coupled. For no coupling, Eq. (16) is a diagonal matrix. However, nonzero expressions for the off-diagonal elements of $M(s)$ indicate an interaction of the power radiated by the corresponding ( $i$ th, $j$ th) modes. In the manner of Yousri and Fahy, ${ }^{20}$ we refer to the diagonal terms as selfradiation resistances and the off-diagonal terms as mutualradiation resistances.

\section{CONTROLLER DESIGN}

A controller was designed using state space realizations for the structural dynamics and radiation dynamics. Next, we outline the steps required to generate the state equations for the radiation dynamics. 


\section{A. Radiation filters}

The first step in constructing the radiation filter is to compute the transfer functions $h_{i}(\theta, \phi, \omega)$ associated with the spatial functions $\Phi_{i}$. For very simple structures, such as the beam used in the next section, this can be done analytically, but, in general, it will have to be done numerically for a sufficiently fine grid in the $(\theta, \phi, \omega)$ space. Then, $M(\omega)$ can be computed from Eq. (16) by numerical quadrature. The matrix $M(s)$ is formed by replacing each element of $M(\omega)$ by a rational Laplace transform that matches the desired frequency response to the specified accuracy.

One way to compute the spectral factorization given by Eq. (20) is to find a state-space realization of the transfer function $M(s)$ and to use the state-space algorithms found in Francis. ${ }^{17}$ This results in a state-space representation of the radiation filter of the form

$$
\begin{aligned}
& \dot{r}=A_{G} r+B_{G} w, \\
& z=C_{G} r+D_{G} w .
\end{aligned}
$$

An alternative approach, which may be numerically easier, is to perform a Cholesky decomposition of the $M(\omega)$ matrices that are known numerically at a given set of frequencies. This gives the values of $G(\omega)$ directly at the given frequencies. Then, $G(s)$ can be computed by fitting rational Laplace transforms to $G(\omega)$, and then a state-space realization of the form shown in Eq. (23) and (24) may be computed.

\section{B. Control law}

Using the state space realization of the radiation filter $G(s)$, we can write the overall system equation as

$$
\left[\begin{array}{c}
\dot{w} \\
\dot{r}
\end{array}\right]=\left[\begin{array}{cc}
A & 0 \\
B_{G} & A_{G}
\end{array}\right]\left[\begin{array}{c}
w \\
r
\end{array}\right]+\left[\begin{array}{c}
B \\
0
\end{array}\right] u,
$$

with a corresponding output equation

$$
z=\left[D_{G} C_{G}\right]\left[\begin{array}{l}
w \\
r
\end{array}\right] .
$$

Assuming that there is a limit to the amount of control energy that can be applied in a given time interval, the cost function is chosen to be

$$
J_{\mathrm{rad}}=\int_{0}^{\infty}\left(z^{T} z+\rho u^{T} u\right) d t .
$$

Minimizing this cost function produces a tradeoff between the total radiated energy and the total control energy used. As $\rho$ approaches zero, minimizing the $\operatorname{cost} J_{\text {rad }}$ is equivalent to minimizing the radiated energy.

The problem we have posed above is in the form of a standard, infinite-time, linear quadratic regulator (LQR) problem. ${ }^{21}$ The optimal control is a time-invariant state feedback of the form

$$
u=K_{w}^{\mathrm{rad}} w+K_{r}^{\mathrm{rad}} r,
$$

where the feedback gains $K_{w}^{\mathrm{rad}}$ and $K_{r}^{\mathrm{rad}}$ are determined from the solution of an algebraic Ricatti equation. If the full state is not available for feedback, the problem can be put in the form of a linear quadratic Gaussian (LQG) problem. The states must be estimated by a Kalman filter and the estimates used in the feedback control law.
TABLE I. Beam physical parameters.

\begin{tabular}{ll}
\hline \hline Young's modulus, $E$ & $2.04 \times 10^{11} \mathrm{~Pa}$ \\
Mass per unit length, $\mathrm{m}$ & $0.491 \mathrm{~kg} / \mathrm{m}$ \\
Length, $L$ & $1.0 \mathrm{~m}$ \\
Width, $b$ & $0.125 \mathrm{~m}$ \\
Thickness, $h$ & $0.5 \mathrm{~mm}$ \\
\hline
\end{tabular}

For purposes of comparison, a cost function of the form

$$
J_{\text {vib }}=\int_{0}^{\infty}\left(w^{T} w+\rho u^{T} u\right) d t
$$

is also considered. Minimizing this cost function produces a tradeoff between total vibrational amplitude and the control energy used. Again, the solution is state feedback and the control law is of the form

$$
u=K_{w}^{\mathrm{vib}} w \text {. }
$$

\section{NUMERICAL EXAMPLE}

To illustrate the ideas discussed in the preceding sections, consider an Euler-Bernoulli model for a uniform bar in a baffled, clamped-clamped configuration:

$$
\begin{aligned}
& E I \frac{\delta^{4} y(x, t)}{\delta x^{4}}+m \frac{\delta^{2} y(x, t)}{\delta t^{2}}=f(x, t), \\
& y(0, t)=y(L, t)=0 ; \quad \frac{\delta y(0, t)}{\delta x}=\frac{\delta y(L, t)}{\delta x}=0,
\end{aligned}
$$

where $y$ is the displacement and $f$ is the force vector containing the disturbance and control actuator inputs. The parameters of the beam are given in Table I. The beam area moment of inertia, $I$, is computed using $I=b h^{3} / 12$.

The mode shapes are given by ${ }^{16}$

$$
\Phi(x)=\cos k_{i} x-\cosh k_{i} x+R_{i}\left(\sin k_{i} x-\sinh k_{i} x\right),
$$

where

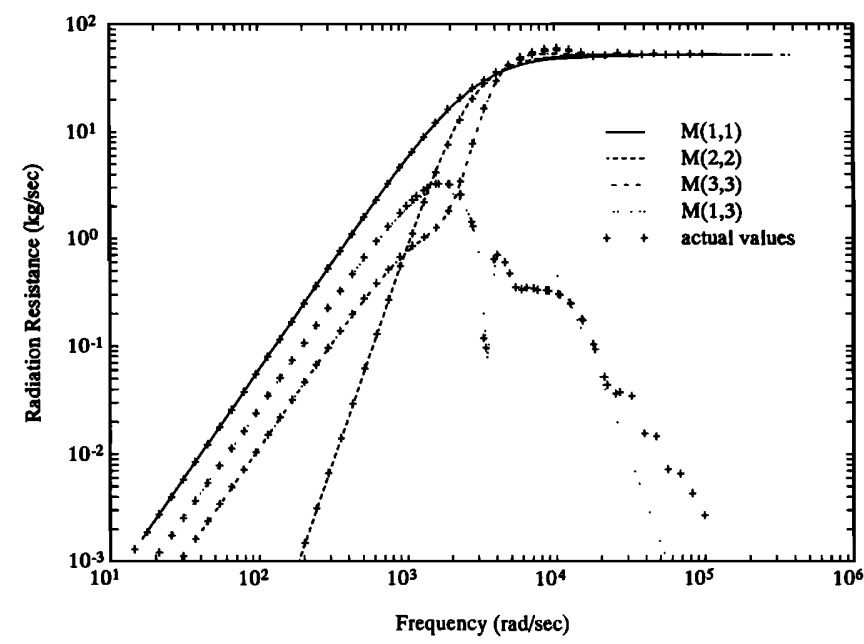

FIG. 1. Numerical representation of $M(\omega)$ compared to polynomial representations. 


$$
R_{i}=\left(\cos k_{i} L-\cosh k_{i} L\right) /\left(\sinh k_{i} L-\sin k_{i} L\right)
$$

and $k_{i} L$ is the $i$ th root of $\cosh k_{i} L \cos k_{i} L=1$. For simplicity, it will be assumed that the structure can be accurately modeled by using the first three modes.

The matrix $M$ was computed numerically at a large number of frequencies using Eq. (16). The integration was performed over a half sphere on one side of the baffle; the results are plotted in Fig. 1. There is no interaction between the radiation from mode 2 and the radiation from modes 1 and 3 . There is interaction between the energy radiated from modes 1 and 3.

These interaction results can be explained in physical terms. For the baffled, clamped-clamped beam, modes 1 and 3 are volumetric modes, while mode 2 is not. At low frequencies $(k L \ll 1)$, the radiation from modes 1 and 3 is due primarily to the volume of fluid displaced. The total volume displaced by the combination of these modes depends on their relative magnitude and phasing, and, hence, the energy radiated by these modes is not the sum of the energies that would be radiated independently. This gives rise to the interaction terms. In terms of pressure patterns, modes 1 and 3 act like monopole sources at low frequencies. Depending on the magnitude and phasing of these modes, the far-field pressures will either enhance or diminish each other over the entire pattern, causing the energy radiated to vary depending on the interaction of the modes. At high frequencies, these arguments do not apply and the interaction terms go to zero.

The pressure pattern of mode 2 resembles a dipole at low frequencies with half of its pattern $180^{\circ}$ out of phase with the other half. Thus, if the superposition of the pressure from mode 2 with the pressure from modes 1 or 3 increases the total pressure over part of the pattern, it will decrease it correspondingly over the other part, resulting in no net change in the total radiated energy. This accounts for the zero interaction terms between mode 2 and modes 1 and 3 .

The direction in which the energy is radiated, however, will depend on the phasing of mode 2 relative to modes 1 and 3. Also, had we only been concerned with radiation into a sector smaller than the half-space, interaction terms between mode 2 and modes 1 and 3 would have appeared in the analysis.

The radiation filter was computed from a rational approximation to $M(s)$. The approximate values of $M(s)$ for $s=j \omega$ are plotted with the desired values, $\boldsymbol{M}(\omega)$, in Fig. 1 and, except for differences in the $(1,3)$ term, the fits are excellent. Figure 2 shows the frequency responses of the elements of the radiation filter and the natural vibration frequencies of the modes are indicated.

A state-space representation for the overall system was constructed as in Eq. (25) and feedback controllers for the acoustic problem [Eq. (27)] and the vibration problem [Eq. (29)] were computed. Computer simulations were run to compare the performance of the two controllers. Because the magnitude of $z^{T} z$ in Eq. (27) is much less than the magnitude of $w^{T} w$ in Eq. (29), the values of $\rho$ in these two

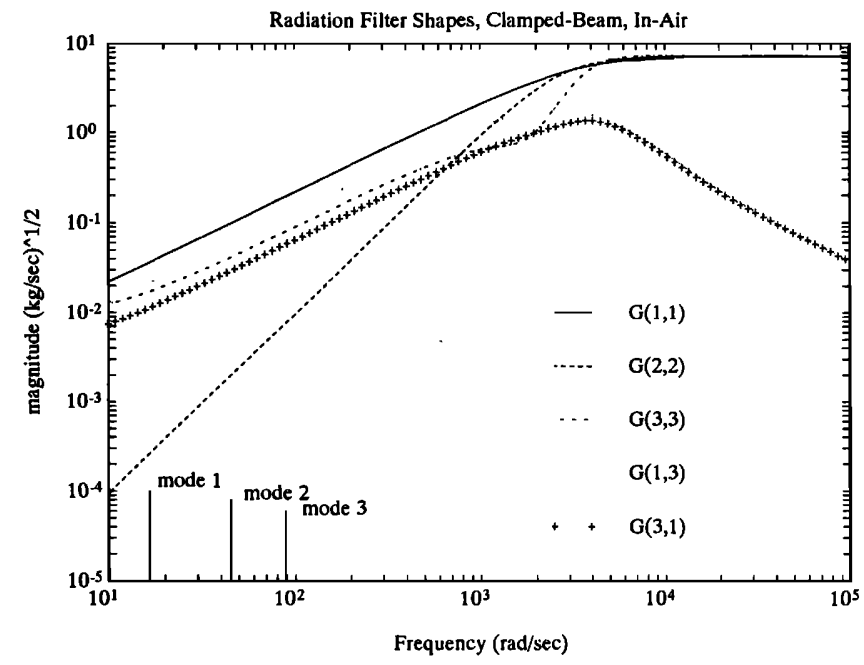

FIG. 2. Radiation filters from factored matrix $G(s)$.

expressions were adjusted on the basis of simulation experiments to make the total energy used by the controllers equal.

The first simulation assumed that there was one actuator located at $x=0.25$ and was initiated by a short-duration pulse on the beam at the same location. The initial conditions of the system were taken to be zero for all states. The time histories of the modal amplitudes using the vibration controller and the acoustic controller are shown in Figs. 3 and 4.

The acoustic controller works harder at suppressing mode 3 than does the vibration controller and leaves mode 2 virtually untouched. This matches our intuition based on radiating efficiencies of the modes. For the time interval considered, the acoustic controller reduced the total energy radiated to the far field by $38 \%$ compared to the vibration controller. Further details of the simulation are summarized in Table II.

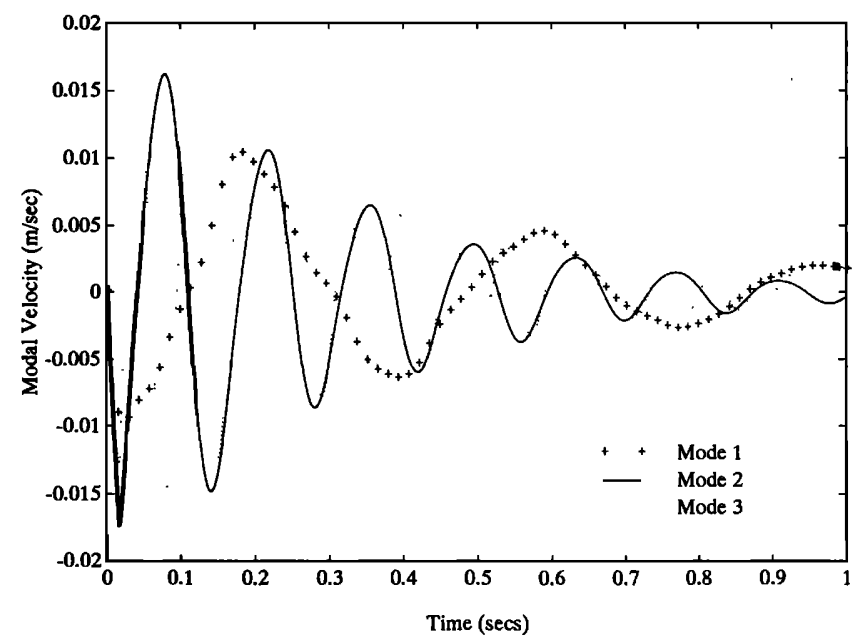

FIG. 3. Modal time histories using vibration control, one actuator at $x=0.25 L$. 


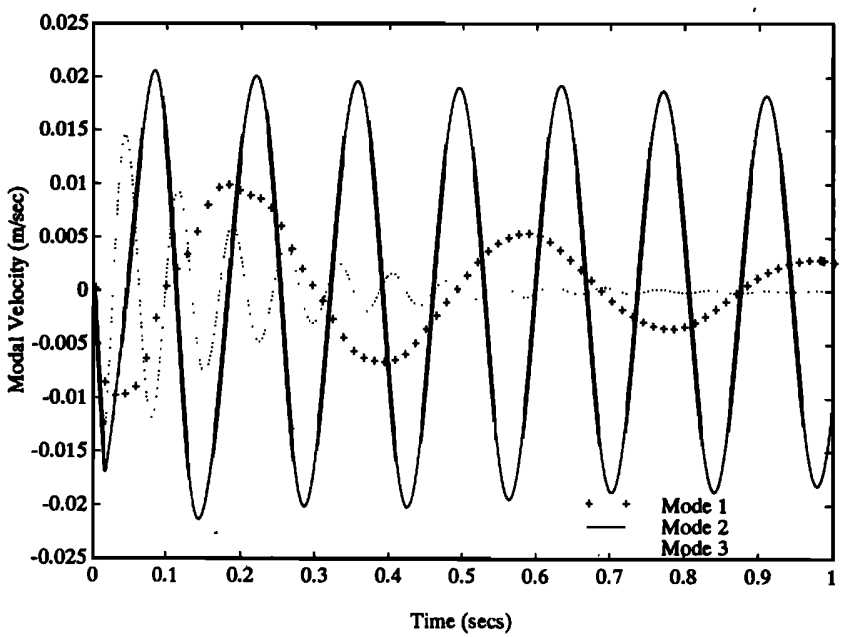

FIG. 4. Modal time histories using acoustic control, one actuator at $x=0.25 L$.

The second simulation assumed that there were two actuators located at $x=0.29$ and $x=0.50$. The short duration pulse was applied at the position $x=0.29$ only. The time histories of the modal amplitudes using the two controllers are shown in Figs. 5 and 6.

The acoustic controller again suppresses the vibration of mode 3 much faster than the vibration controller and leaves mode 2 virtually untouched. Also, it does not allow mode 1 to oscillate, but forces it to decay exponentially. This reduces the radiation from this mode and is physically possible due to the extreme flexibility of the structure. For the time interval considered, the acoustic controller reduced the total energy radiated by $69 \%$ compared to the vibration controller. Both controllers used more energy than in the first case, as can be seen by the increased damping of the modal amplitudes, but this resulted in an even greater advantage for the acoustic controller.

\section{CONCLUSION}

The major result of this paper is a method for estimating, in real time, the total acoustic energy radiated to the far field from measurements on the structure. This was accomplished by first spatially decomposing the structure, using the $\Phi_{i}$ functions, and then decomposing the radiation of these spatial functions with respect to temporal frequency.

These frequency response functions were used to construct a radiation filter. To estimate the radiation, velocity measurements of the structural vibration are converted into velocities of the predetermined spatial functions and these

TABLE II. Simulation results.

\begin{tabular}{lccc}
\hline \hline Control objective & Number of actuators & $\rho$ & Acoustic energy \\
\hline Vibration & 1 & $4.5 e-2$ & $4.32 e-7$ \\
Acoustic & 1 & $1.0 e-4$ & $2.68 e-7$ \\
Vibration & 2 & $4.5 e-3$ & $1.93 e-7$ \\
Acoustic & 2 & $5.0 e-4$ & $6.04 e-8$ \\
\hline \hline
\end{tabular}

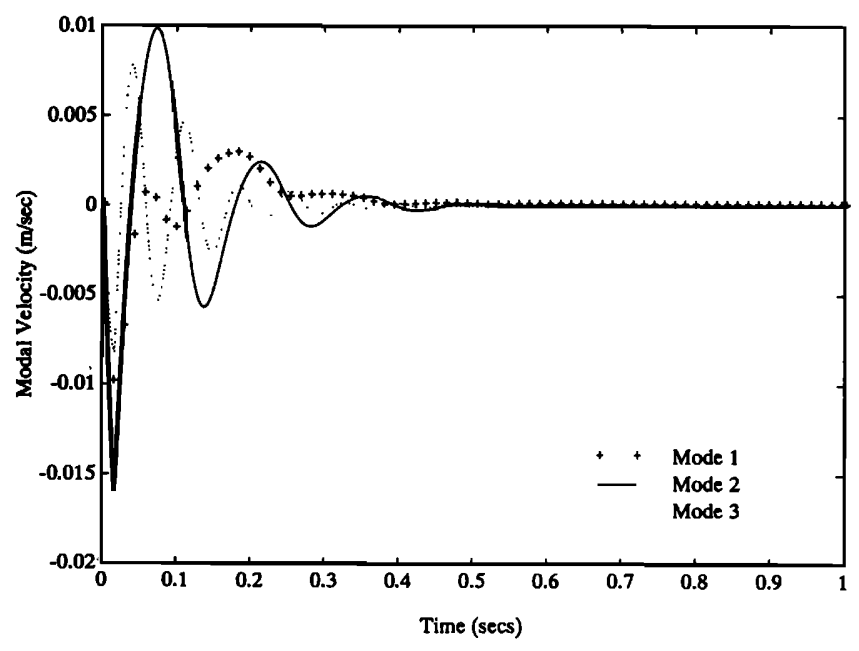

FIG. 5. Modal time histories using vibration control, two actuators at $x=0.29 L, x=0.5 L$.

velocities are filtered to produce an output signal whose integrated square is proportional to the radiation.

This is in contrast to recent work by Borgiotti ${ }^{22}$ and Photiadis, ${ }^{23}$ where the system is first decomposed temporally, by considering a fixed frequency of excitation, and then decomposed spatially into orthogonal spatial functions that radiate efficiently and those that radiate inefficiently. Thus they arrive at spatial filters that can be used to determine the amount of far-field radiation. To use this approach for transient or broadband persistent excitations, however, would seem to require that the excitation first be decomposed into temporal harmonics.

The radiation filter developed in this paper was incorporated into the design of a controller to minimize acoustic radiation. Several computer simulations have been presented to demonstrate the effectiveness of this acoustic controller when compared to a vibration controller using the same control energy. Further work is under way to explore the degree to which the radiation filters can be reduced in complexity without significant loss of performance.

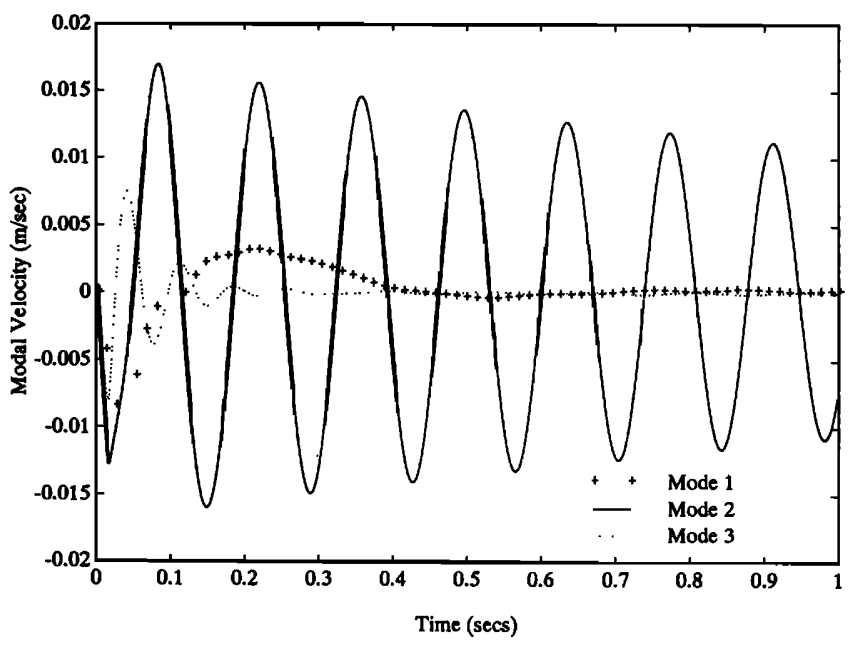

FIG. 6. Modal time histories using acoustic control, two actuators at $x=0.29 L, x=0.5 L$. 


\section{ACKNOWLEDGMENT}

This work was supported by ONR Contract N0001488-K-0721.

${ }^{1}$ H. H. Robertshaw, R. H. Wynn, Jr., S. L. Hendricks, and W. W. Clark, "Dynamics and control of a spatial active truss actuator," in Proceedings of the 30th SDM Conference, April 1989, AIAA Paper No. 89-1328-CP.

${ }^{2} \mathrm{~L}$. Meirovitch, H. Baruh, and H. Oz, "A comparison of control techniques for large flexible structures," J. Guidance Contr. Dynam. 6(4), 302-310 (1983).

${ }^{3}$ A. Baz and S. Poh, "Performance of an active control system with piezo actuators," J. Sound Vib. 126(2), 327-343 (1988).

$4 \mathrm{~J}$. S. Burdess and A. V. Metcalfe, "Active control of forced harmonic vibration in finite degree of freedom structures with negligible damping," J. Sound Vib. 91(3), 447-459 (1983).

${ }^{5} \mathrm{M}$. Balas, "Active Control of Flexible Systems," J. Optimization Theor. Appl. 25, 415-436 (1978).

${ }^{6} \mathbf{P}$. Hagedorn and J. T. Schmidt, "Active vibration damping of flexible structures using the travelling wave approach," in Proceedings of the Second International Symposium on Spacecraft Flight Dynamics, Darmstadt, October, 1986 (ESA SP-255, December 1986).

${ }^{7}$ B. R. Mace, "Active control of flexural vibrations," J. Sound Vib. 114(2), 253-270 (1987).

${ }^{B}$ A. H. von Flotow and B. Schafer, "Wave absorbing controller for a flexible system," J. Guidance Contr. Dynam. 9, 673-680 (1986).

${ }^{9}$ C. R. Fuller, R. J. Silcox, V. L. Metcalf, and D. E. Brown, "Experiments on structural control of sound transmitted through an elastic plate," Proceedings of American Control Conference, 2079-2084, Pittsburgh, PA (1989).

${ }^{10} \mathrm{C}$. R. Fuller, "Active control of sound transmission/radiation from elastic plates by vibration inputs: Analysis," J. Sound Vib. 136(1), 1-15 (1990).
${ }^{11}$ B. Widrow and S. D. Stearns, Adaptive Signal Processing (Prentice-Hall, Englewood Cliffs, NJ, 1985).

${ }^{12}$ S. J. Elliott, I. M. Stothers, and P. A. Nelson, "A multiple error LMS algorithm and its application to the active control of sound and vibration," IEEE Trans. Acoust. Speech Signal Process. ASSP-35(10), 14231434 (1987).

${ }^{13}$ L. Meirovitch and S. Thangitham, "Active control of sound radiation pressure," ASME J. Vib. Acoust. 112, 237-244 (1990).

${ }^{14}$ C. R. Fuller, C. A. Rogers, and H. H. Robertshaw, "Active structural acoustic control with smart structures," in Proceedings of the SPIE, Vol. 1170: Fiber Optic Smart Structures and Skins, Boston, MA (September 1989), pp. 338-358.

${ }^{15}$ J. A. Mann, J. Tichy, and A. J. Romano, "Instantaneous and time-averaged energy transfer in acoustic fields," J. Acoust. Soc. Am. 82, 17-30 (1987).

${ }^{16}$ D. C. Youla, "On the factorization of rational matrices," IRE Trans. Inf. Theory 172-189 (1961).

${ }^{17}$ B. Francis, $A$ Course in $H_{\infty}$ Control Theory (Springer-Verlag, New York, 1986).

${ }^{18}$ B. P. Lathi, Signals, Systems and Communication (Wiley, New York, 1965).

${ }^{19}$ T. Kailath, Lectures on Linear Least-Squares Estimation (Springer-Verlag, New York, 1976).

${ }^{20} \mathrm{~S}$. Yousri and F. Fahy, "Acoustic Radiation by unbaffled cylindrical beams in multimodal transverse vibration," J. Sound Vib. 40(3), 299-306 (1975).

${ }^{21}$ D. E. Kirk, Optimal Control Theory: An Introduction (Prentice-Hall, Englewood Cliffs, NJ, 1970).

${ }^{22} \mathrm{G}$. Borgiotti, "The power radiated by a vibrating body in an acoustic fluid and its determination from boundary measurements," J. Acoust. Soc. Am 88, 199-209 (1990).

${ }^{23} \mathrm{D}$. Photiadis, "The relationship of singular value decomposition to wavevector filtering in sound radiation problems," J. Acoust. Soc. Am 88, 1152-1159 (1990). 